\title{
Left Lumbar Subcutaneous Cold Abscess with Tuberculous Spondylitis
}

\author{
Atsuko YUKi, Toshinori TANAKA, Yoshifumi KIYONO*, Akihiro TsuKADA*, \\ Mineko OHIRA, Kiyoshi OHIRA and Yoshio OKUBO
}

\begin{abstract}
We report a 29-year-old man with a rare left lumbar subcutaneous cold abscess complicated by tuberculous spondylitis during the treatment of pulmonary tuberculosis. Pulmonary tuberculosis was rapidly improved by anti-tuberculous drugs, however curative operation for tuberculous spondylitis was necessary after 18 months because tuberculous spondylitis was overlooked. Imaging techniques are important in helping to establish a diagnosis of tuberculous spondylitis. It should be stressed that a high clinical index of suspicion for tuberculosis is needed to correctly diagnose this disease.
\end{abstract}

(Internal Medicine 43: 319-322, 2004)

Key words: tuberculous spondylitis, cold abscess, magnetic resonance imaging

\section{Introduction}

The incidence of all forms of tuberculosis, including skeletal infection, has markedly declined in developed countries during the past several decades. In Japan, the mortality rate, incidence, and prevalence of tuberculosis have decreased remarkably. However, in 1997 the number of newly registered patients increased for the first time and then the ministry of Health, Labour and Welfare announced a strong warning regarding the increased incidence in 1999. The increased number of tuberculosis cases was thought to be due to the increase in the older population and number of acquired immune deficiency syndrome patients. Even under the announcement, there are some problems in tuberculosis such as the delay in detecting and treating tuberculosis in Japan.

We report a male patient with left lumbar subcutaneous cold abscess complicated by tuberculous spondylitis. The patient was diagnosed as having pulmonary tuberculosis with left pleural effusion; it was improved rapidly by the treatment of anti-tuberculous drugs. However tuberculous spondylitis was overlooked despite complaints of back pain and magnetic resonance imaging (MRI) studies. Thereafter, left lumbar subcutaneous cold abscess developed. Tuberculous spondylitis was not improved until curative surgery was performed.

\section{Case Report}

A 29-year-old Japanese man complaining of dyspnea, cough, night sweats with high grade fever $\left(39^{\circ} \mathrm{C}\right)$ was admitted to hospital on March 13, 2001.

He had a 10-month history of cough and low back pain. He was referred to hospital and underwent MRI of the spine on May 18, 2000. However, abnormal findings of L1 and L2 vertebral bodies were overlooked (Fig. 1A, B) and nonsteroidal anti-inflammatory drug (NSAID) was administered. $\mathrm{He}$ had received the BCG vaccine in childhood, and tuberculin reaction on admission showed a $19 \times 18 \mathrm{~mm}$ area of reddening, without induration. Lymph nodes in the neck were not palpable. The breath sound on the left lower back was distant. The chest roentgenogram showed a cavity with an infiltrative shadow and small nodular shadow in the left upper lung field and massive left pleural effusion (Fig. 2A). Laboratory findings showed leukocyte count $\left(6,700 / \mathrm{mm}^{3}\right.$ with $15.9 \%$ lymphocytes), hemoglobin $(12.6 \mathrm{~g} / \mathrm{dl})$, erythrocyte sedimentation rate (ESR) $(42 \mathrm{~mm} / \mathrm{h}), \mathrm{C}$-reactive protein (CRP) $(3.3 \mathrm{mg} / \mathrm{dl})$, and negative human immunodeficiency virus (HIV) antibody. Pleurocentesis was performed, and a yellow colored pleural effusion was obtained that showed 5.0 $\mathrm{g} / \mathrm{dl}$ of total protein, and $37.6 \mathrm{U} / \mathrm{l}$ of adenosine deaminase activity. Staining of sputum and gastric juice for acid-fast bacilli as well as polymerase chain reaction (PCR) for Mycobacterium tuberculosis of sputum and gastric juice were all

From the Department of Internal Medicine and *the Department of Orthopedics, Higasinagano National Hospital, Nagano

Received for publication July 9, 2003; Accepted for publication December 3, 2003

Reprint requests should be addressed to Dr. Yoshio Okubo, the Department of Internal Medicine, Higasinagano National Hospital, 2-477 Uwano, Nagano 381-8567 


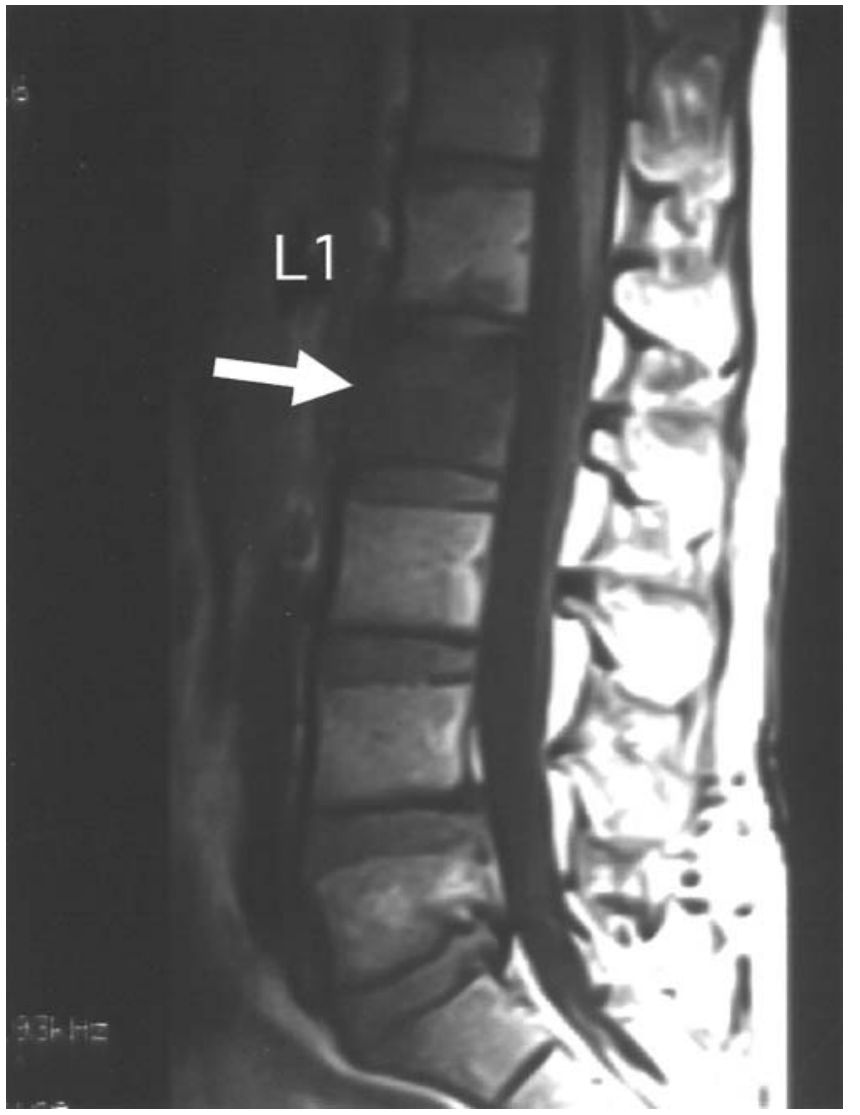

A

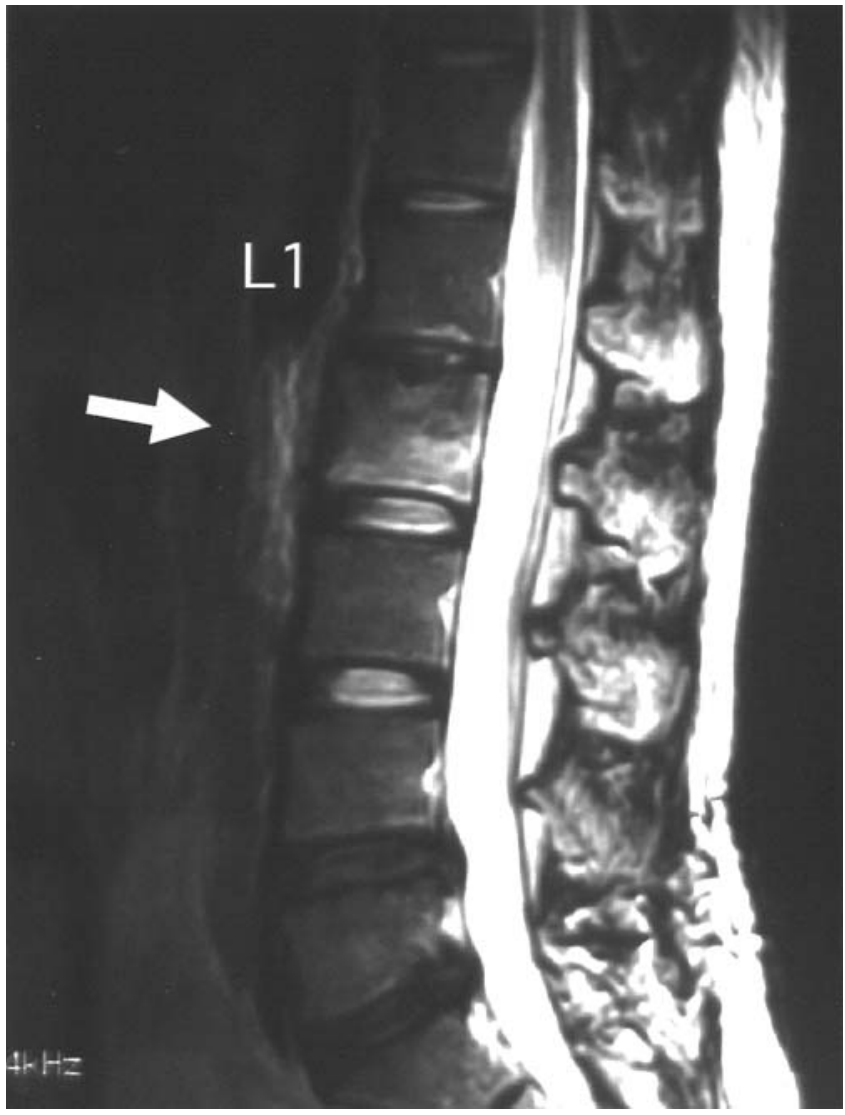

B

Figure 1. A) MRI spin echo mid-sagittal T1-weighted image showed destruction of L1 and L2 vertebral bodies (arrow). B) A paravertebral anterior abnormal soft tissue component was seen on sagittal T2-weighted image (arrow).

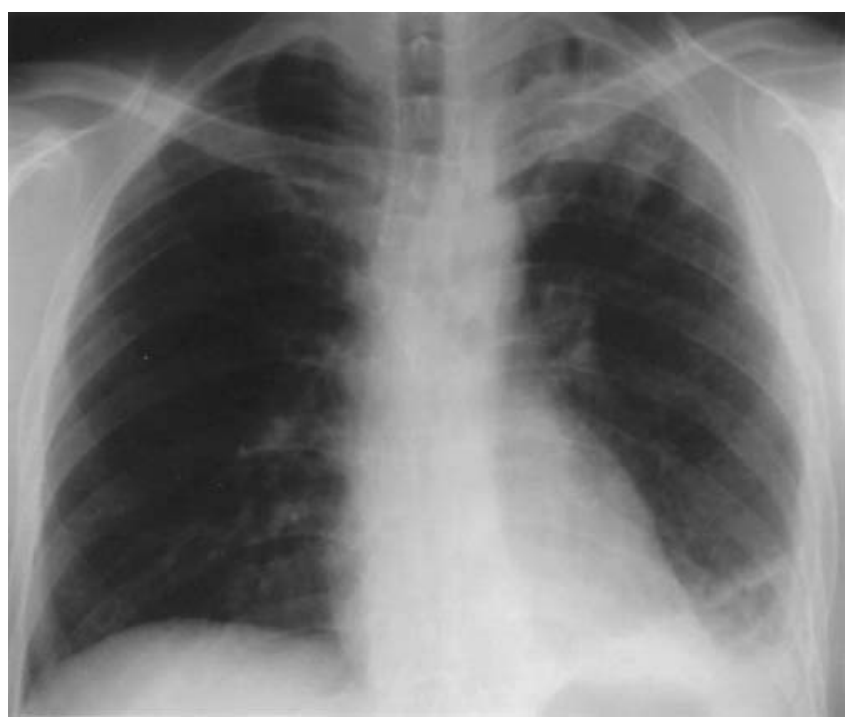

A

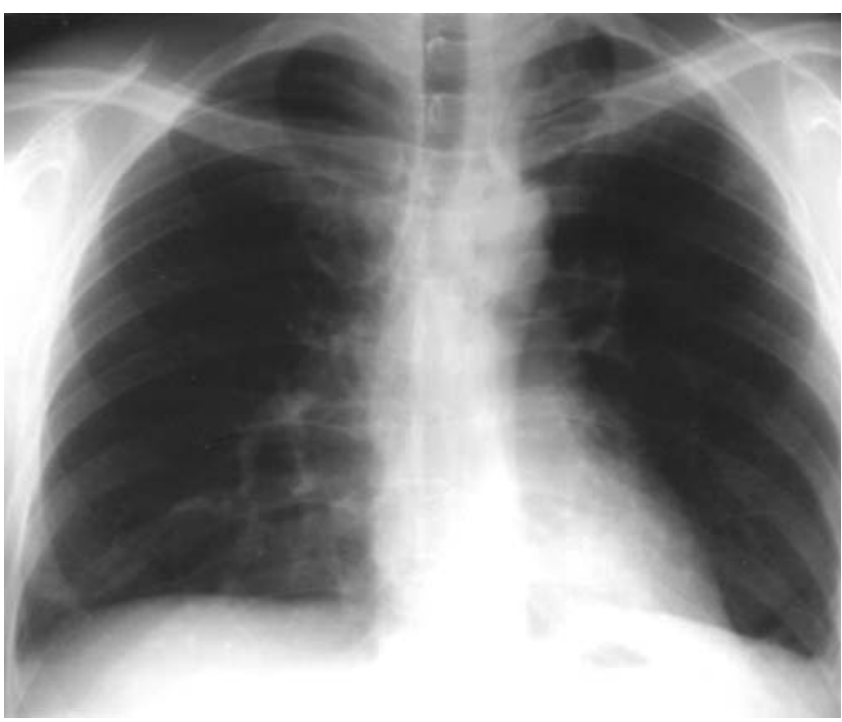

B

Figure 2. A) Chest roentgenogram on admission. A cavity with an infiltrate shadow in the left upper lung field and massive left pleural effusion were observed. B) Chest roentgenogram after the treatment of anti-tuberculous drugs for 5 months. The infiltrate shadow and pleural effusion had disappeared. 


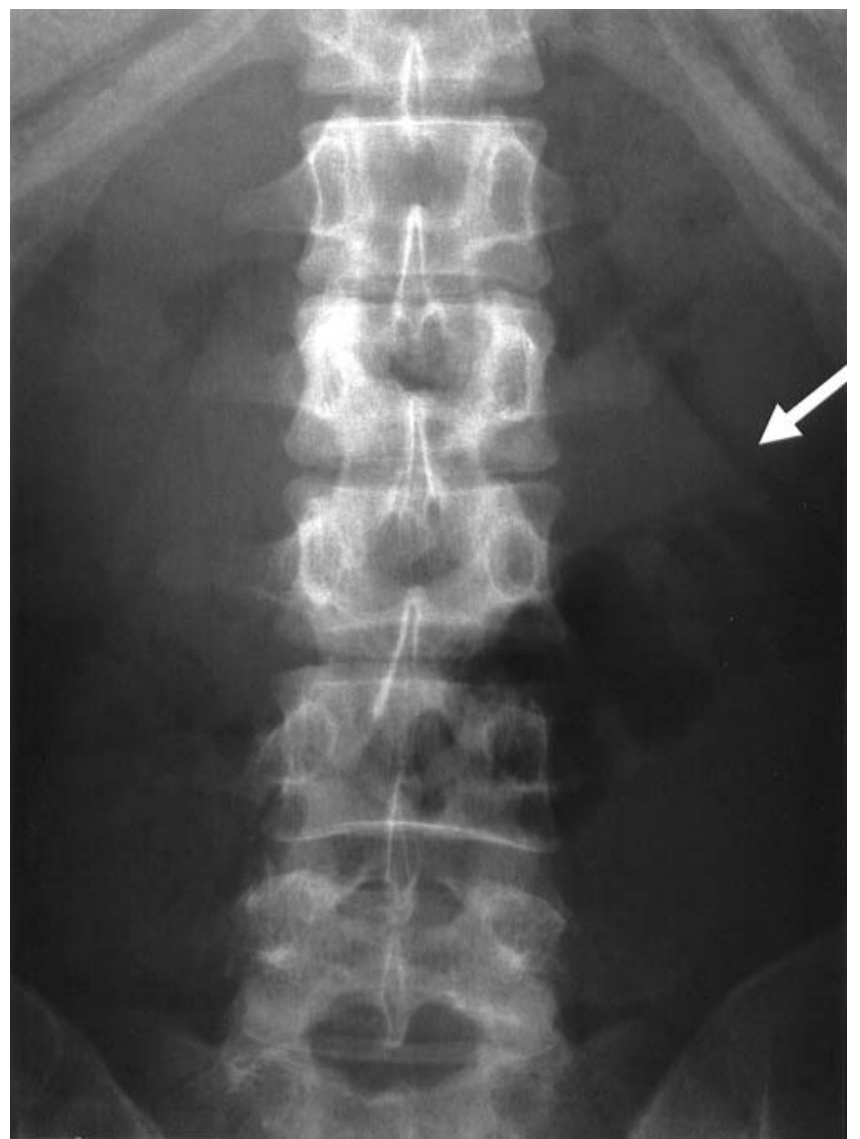

Figure 3. Abdominal roentgenogram showed an abnormal left psoas shadow (arrow).

positive. Mycobacterium tuberculosis was observed on an 8 -week culture of pleural effusion. He was administered antituberculous drugs (pirazinamide (PZA) $1.2 \mathrm{~g} /$ day, isoniazid (INH) $400 \mathrm{mg} /$ day, rifampicin (RFP) $450 \mathrm{mg} / \mathrm{day}$, and streptomycin sulfate (SM) $0.75 \mathrm{~g} / \mathrm{day})$. After 2 weeks of chemotherapy, he noticed low back pain again, and an abnormal left psoas shadow was found on plain abdominal roentgenogram (Fig. 3). Six weeks later, ethambutol hydrochloride (EB) $750 \mathrm{mg} /$ day was administered instead of SM because SM resistance was identified. On July 25, 2001 the levels of ESR and CRP became within the normal range. Infiltrated shadow on chest roentgenogram was improved and pleural effusion disappeared (Fig. 2B), then he was discharged. On October 30, 2001 he found a large fluctuating swollen mass on his left lower back. There were no neurological deficiencies. On November 13, abdominal computed tomography (CT) suggested a cold abscess in the left psoas muscle (Fig. 4) with destruction of the L1, L2, L3 and L4 vertebral bodies. Active tuberculous spondylitis and cold abscess in the left low back were suggested by clinical and radiographic findings. On January 14, 2002, curative surgery for tuberculous spondylitis was performed. Necrotic bone was resected and white pus $(970 \mathrm{ml})$ was drained (Fig. 5).

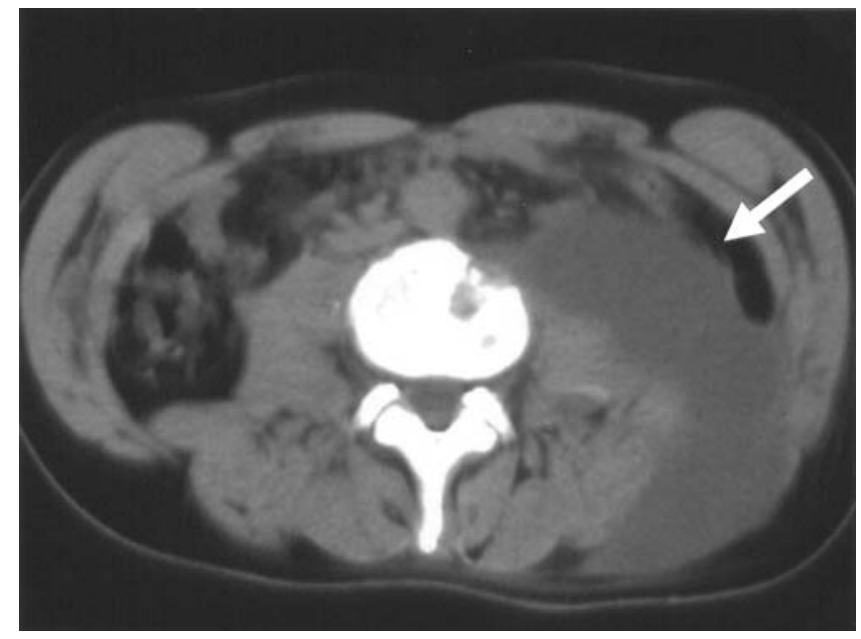

Figure 4. Abdominal computed tomography showed a large paravertebral soft tissue mass (arrow), suggesting a cold abscess in the left psoas muscle.

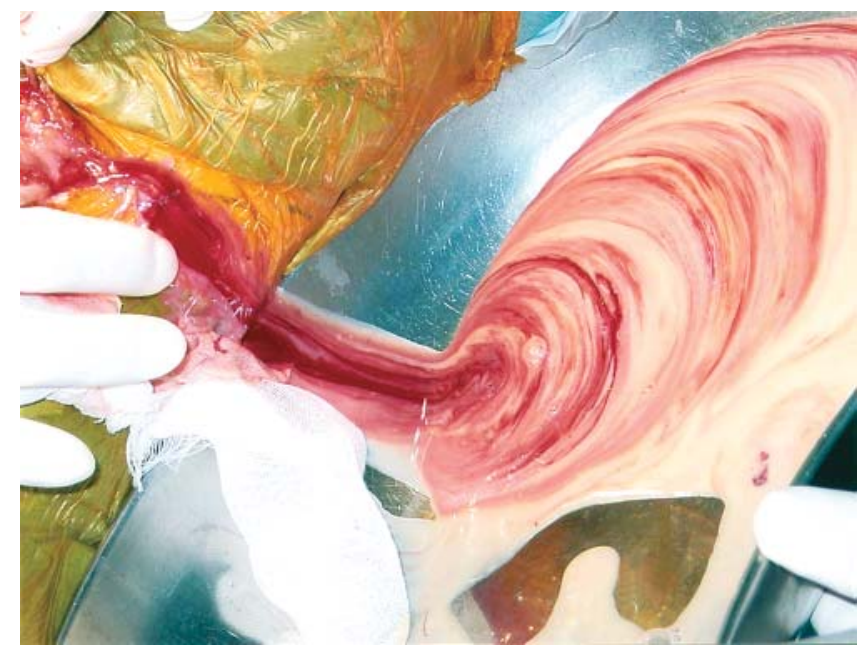

Figure 5. White pus was spontaneously drawn when the peritoneum was opened.

Neither staining nor culture for acid-fast bacilli were observed in cold abscess. Furthermore, there were no pathological findings such as caseous necrosis with Langhan's giant cells and epithelium cells in the resected bone. Definitive diagnosis depending on histological examination may be difficult to obtain (1). In this case, diagnosis of tuberculous spondylitis was based on clinical symptoms and radiological findings. Then, INH, RFP and PZA were further administered for 12 months and kanamycin sulfate (KM) 1.0 g/day was newly administered for 6 months, because side effect of EB occurred. Six months later after the curative surgery, he was discharged. Currently the patient is in good condition. 


\section{Discussion}

It is reported that tuberculosis manifests itself in extrapulmonary organs in about $15 \%$ of cases (2). Skeletal tuberculosis occurs in $1 \%$ of all tuberculosis (3), and the spine is involved in approximately one-half of these cases. Thoracic, thoraco-lumbar and lumbar vertebrae are the most frequently involved, while cervical and sacral involvement is rare $(2,4)$. The prevalence of tuberculous spondylitis is gradually increasing in many industrialized countries (5). One of the reasons for resurgent tuberculosis is thought to be due to epidemic HIV infection (6). The other cause is due to the homeless and expanding immigrant population $(7,8)$. The present patient had been employed in the service industry and working hard from early morning to late night without eating regular meals nor getting enough sleep. This kind of modern lifestyle seems to be one reason for the increasing susceptibility to tuberculosis in Japan (9). It is necessary to take a history of the patient's daily life in detail to make a correct diagnosis.

The mechanism of spinal tuberculosis is first implantation of mycobacteria in the vertebral body and then the development of osteomyelitis. Classically, the case of two adjacent vertebrae with disc involvement is due to haematogenous spread of organisms through one arteria intravertebralis feeding the lower half of one vertebrae and the upper half of the adjacent vertebrae. After bone infection, paraspinal abscess develops as the pus moves up and down in the spine $(3,4)$.

Common complaints in spinal tuberculosis include localized back pain, low grade fever and weight loss. Physical examination often demonstrates tenderness over the affected region. In more advanced cases, neurological symptoms and spinal deformity are present. Extension of infection to adjacent structures; the pleura, retropharyngeal areas, skin, psoas and the viscera, is common (1). Laboratory data such as elevated ESR, a high level of CRP and positive tuberculin skin test provide some information, but are by themselves not sufficient to establish a firm diagnosis.

However, imaging techniques are important in helping to establish the diagnosis. Conventional roentgenogram demonstrates a destructive process in the vertebrae, involvement of the disc space, and spinal deformity (10), and abnormal psoas shadow. Furthermore, CT and MRI provide a great advantage in the differential diagnosis of tuberculous spondylitis. MRI is a more useful apparatus to clearly demonstrate paravertebral abscess and is also more useful in the differential diagnosis of vertebral osteomyelitis and disk space infection than CT (11). In the present case, MRI of the spine demonstrated abnormal findings of L1 and L2 vertebral bodies. However failure to observe these findings induced an 18-month delay in the diagnosis of tuberculous spondylitis. Due to the delayed finding, he was administered PZA, INH, RFP as well as KM for a prolonged period. It is thought that it was difficult to maintain an effective concentration of tuberculous drugs in the local lesion preoperatively.

We report a case of tuberculous spondylitis which is now an uncommon occurrence in Japan. If the patient with pulmonary tuberculosis has back pain, the possibility of tuberculous spondylitis that can be rarely encountered must be considered for the correct diagnosis.

\section{References}

1) Alame T, Dierckx P, Ninane V, Sergysels R. Spinal tuberculosis: a report of five cases and a review. Monaldi Arch Chest Dis 51: 5, 362368, 1996.

2) Seaton A, Seaton D, Leitch AG. Crofton \& Douglas's Respiratory Diseases. Blackwell, Oxford, 1989: 395-422.

3) Davidson PT, Horowitz I. Skeletal tuberculosis. A review with patient presentations and discussion. Am J Med 48: 77-84, 1970.

4) Gorse GJ, Pais MJ, Kusske JA, Cesario TC. Tuberculous spondylitis: a report of six cases and a review of the literature. Medicine (Baltimore) 62: 178-193, 1983.

5) Okuyama Y, Nakaoka Y, Kimoto K, Ozasa K. Tuberculous spondylitis (Pott's Disease) with bilateral pleural effusion. Intern Med 35: 883$885,1996$.

6) Shafer RW, Kim DS, Weiss JP, Quale JM. Extrapulmonary tuberculosis in patients with human immunodeficiency virus infection. Medicine (Baltimore) 70: 384-397, 1991.

7) Yamanaka K, Kondo T, Miyao M. Tuberculosis among the homeless people of Nagoya, Japan. Respir Med 88: 763-769, 1994.

8) Shanley DJ. Tuberculosis of the spine: imaging features. AJR Am J Roentgenol 164: 659-664, 1995.

9) Mori T. Present status of tuberculosis in Japan: Rinshou Kagaku 35: 275-281, 1999 (in Japanese).

10) William NR, Stuart MG. Tuberculosis. Little, Brown and Company, Boston New York Toronto London, 1995: 623-633.

11) Paushter DM, Modic MT, Masaryk TJ. Magnetic resonance imaging of the spine: applications and limitations. Radiol Clin North Am 23: 551562,1985 . 